\title{
Power-Cost-Effective Node Architecture for Light-Tree Routing in WDM Networks
}

\author{
G. M. Fernández, Student Member, IEEE, D. Larrabeiti, Member, IEEE, C. Vázquez, Senior, IEEE, \\ and P. C. Lallana
}

\begin{abstract}
We present a novel cost-effective multicast capable optical cross connect (MC-OXC) node architecture which improves efficiency of optical power by constraining splitting to only two output ports, in order to reduce power losses derived from splitting into more than two output ports. This node would manage the following actions when necessary: (a) tap and binarysplitting, which consists of tapping a small percentage of the signal power to the local node (4-8\%) and an $n$-splitting action $(n=2)$; and (b) tap-and-continue. We call this type of node 2-STC node (binary-split-tap-continue). We compare it with other well known state-of-art proposals and analyze its benefits in terms of number of devices and power losses. An evaluation of applicability is given, showing that the binary-split restriction shows a good trade-off between power losses, bandwidth consumption and architectural simplicity. We conclude that the 2-STC node improves power efficiency and contributes to get a good trade-off between use of resources and optical power.
\end{abstract}

Index Terms - OXC node architectures, light-trees, binarysplitting

\section{INTRODUCTION}

$\mathrm{T}$ here are many data applications for WDM (wavelengthdivision multiplexing) networks that require point to multi-point connections, such as GRID computing, videoconferencing, video-on-demand (VOD), high-definition television (HDTV), multimedia document distribution, interactive distance learning, optical storage area networks (OSAN), etc. These demands are known as multicast requests, in which a single source node sends data to a set of destinations [1]. In order to realize all-optical multicast operations, the lightpath concept (a lightpath is an all-optical point-to-point path) has been generalized in [2] to that of light-tree, which consists of an all-optical point-to-multipoint channel originated at any source and has more than one destination node. A light-tree reach several nodes, it is independent regarding to bit-rate, protocol and format [3], and it does not need per-hop packet forwarding. The main element of lighttrees is the optical multicast capability of optical switches, and

Manuscript received March 31, 2008.

G. M. Fernández and D. Larrabeiti are with the Telematics Engineering Department, Universidad Carlos III de Madrid, Spain (phone: +34 647505716; e-mail: gfernandezdc@inv.it.uc3m.es/gfernandezdc@ucsp.edu.pe and dlarra@it.uc3m.es).

C. Vázquez, and P.C. Lallana are with the Electronics Engineering Department, Universidad Carlos III de Madrid, Madrid, Spain (e-mail: cvazquez@ing.uc3m.es and pcontrer@ing.uc3m.es). some node multicast-capable nodes have been proposed. This paper studies these proposals and presents a novel hybrid node architecture that tries to combine the actions of tapping and splitting.

\section{DATA Plane AND CONTROL Plane OF OpticAL MULTICAST}

\section{A. Data Plane: Multicast OXC Node Architectures}

\section{SaD-based MC-OXC Node Architectures}

The split-and-delivery switch ( $\mathrm{SaD}$ switch) is a device that was first proposed in [4], which is able to split an input optical signal into $n$ outputs. This is the main component of the SaDbased multicast-capable node. In order to reduce cost and to improve power efficiency, it was later modified [5]. An alloptical multicast-capable OXC node (MC-OXC) would be mainly constructed with passive optical light splitters in order to enable multicasting. A passive splitter divides an optical signal power into several optical signals without any knowledge about the optical features of the input signal and without changing any property of it except the power [1]. Obviously, a split operation generates power losses, and ideally- the power of each output is the $(1 / n)$-th part of the input.

In [6], an improvement for the SaD-switch was proposed by utilizing configurable splitters instead of passive splitters, which can be instructed to split the incoming signal into $m$ outputs ( $m=1, \ldots, P$ ), where $m=1$ corresponds to no power splitting (a single switch operation), and $m=P$ to a broadcast operation. After the splitting phase, each of the $m$ signals that were split are switched to a given output port by using a $P^{2}$ photonic switch matrix made up of optical switching elements. In the SaD switch design, a large number of optical amplifiers are needed $\left(P^{2}\right)$, which are located after the configurable splitters in order to compensate power losses. These devices amplify optical signals without OEO conversion, and have some drawbacks (high cost, complexity and amplification of noise levels).

Some SaD-based node architectures were proposed [4], that contain $W$ SaD switches ( $W=$ number of wavelengths). Each input fiber is demultiplexed to extract the individual wavelengths, which are directed to their correspondent SaD switches, where splitting and space switching operations are performed. At last, $P$ multiplexers combine the $W$ signals onto 
their corresponding outgoing fibers. Another SaD-based node architecture [4] includes wavelength conversion capability. By the other hand, other architectures hve been proposed to save more power, such as MOSAD [5], which uses the splittingsharing concept. In this, all requests share one single power splitter, therefore only one multicast request can be attended at a time. It reduces costs and complexity, however it causes a high blocking performance.

\section{Tap-and-Continue MC-OXC Node Architecture}

We have to point out that SaD-based nodes have a severe problem in which much power loss is wasted: if the local node is a member of the multicast request, one of the split outputs are dropped locally [5]. With the aim to solve this obstacle and reduce the cost, an architecture called Tap-and-Continue $(\mathrm{TaC})$ was proposed in [7]. In this approach, when the node itself is a member of the multipoint connection, only a very small fraction $(0.5 \%-10 \%)$ of the incoming signal power is tapped and the rest continues to any output port. This node contains a $P \times(P+1)$ wavelength routing switching (WRS) module per every wavelength and one shared tap-andcontinue module (TCM), which taps to the local node. Authors have proven that any multicast session can be realized by using only $\mathrm{TaC}$ nodes, and some multicast routing algorithms for TaC-networks have been proposed [5] [8].

\section{B. Control Plane: MC-RWA and Power Loss Constraints}

Regarding to control plane, the main problem to solve is the multicast routing and wavelength assignment (MC-RWA) problem. Finding the optimal light-tree is an NP-complete problem and is formalized as the Steiner tree problem [9]. There is plenty of work for the MC-RWA problem, and proposed solutions are designed for one single multicast request, multiple static multicast requests, and multiple dynamic multicast requests. A wide state of the art is presented in [1], and most work is focused on reducing the number of links (bandwidth), wavelengths used, and distances from the source node to every destination node.

MC-RWA has also been treated in optical networks with sparse light splitting (networks with a few multicast-capable nodes). In this case it could be not possible to find a light-tree, and different approaches were made, such as finding a light forest (set of light-trees) that satisfies a multicast request. Also, an algorithm using only TaC-OXC nodes was proposed, and authors showed that any multicast request can be served using only TaC nodes [10].

The splitting of an optical signal implies a considerable power loss that leads to a bound to the times a signal can be split. Thus, since optical amplification increases noise levels, there is a limit on the number of times a signal can be split [5][11]. There are also losses caused by power attenuation along a fiber link. The problem of building light-trees under optical power constraints [6][11][12] is not the focus of this paper. A balanced light-tree would be the optimal solution in order to obtain the best performance of a power-constrained network, and SaD-based nodes would be able to achieve that optimal configuration. In TaC nodes the problem is reduced to the traveling salesman problem.

In this paper, we propose to limit the split fan-out to two branches, and combine it with tapping. This is another variant of the MC-RWA problem, in which the target is to build binary trees as balanced as possible to maximize the number of nodes that receive enough power from tapping.

\section{THE NOVEL BINARY-SPLIT-TAP-CONTINUE NODE ARCHITECTURE}

\section{A. General Node Architecture}

Fig. 2 depicts the general architecture for the binary-splittap-continue node (2-STC node) that is able to:

-- Tap-and-continue: Besides switching, it taps a small fraction of the input power to the local node. Unlike TaC nodes [7], tap is fixed and always performed. TaC nodes need much more optical switches because of the optional tap.

-- Tap-and-binary-split: The node should tap and perform binary-split for multiple requests in a strictly non-blocking manner (also note that tap is always performed).

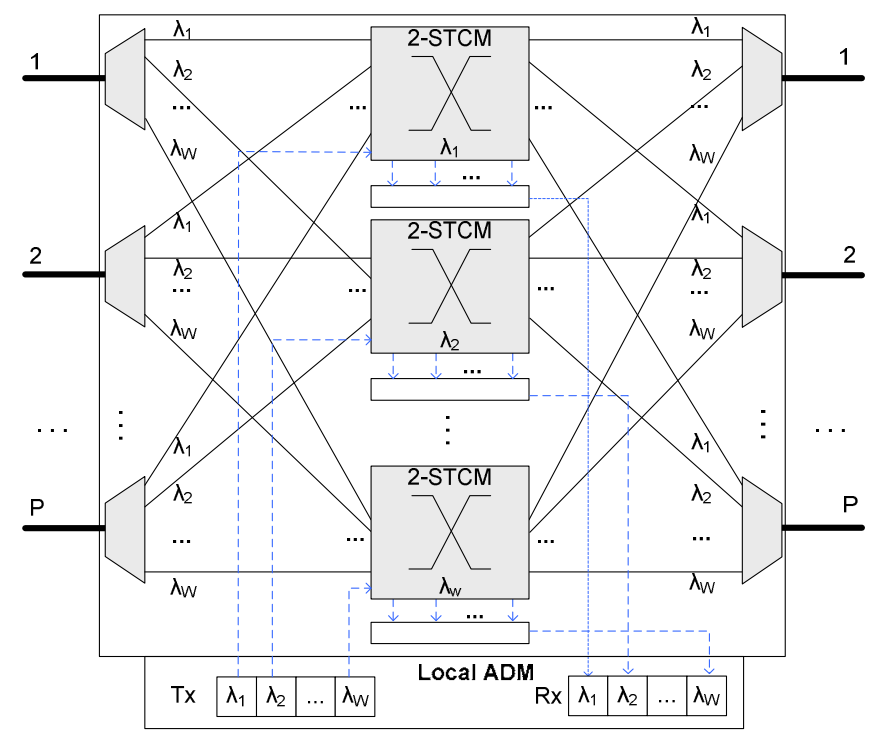

Fig. 2. General architecture of a P x P 2-STC MC-OXC node.

The general architecture is similar to that of the SaD-based node [4][5], but instead of SaD switches, novel Binary-SplitTap-Continue Modules (2-STCM) are used (explained later). It employs $P$ multiplexers to extract individual wavelengths from each input fiber and $P$ demultiplexers to combine individual wavelengths onto the output fibers. An array to receive taps is used $\left(R_{x}\right)$ at the ADM local station, and it could be required a selector in every 2-STCM to choose the correct signal tapped.

\section{B. The Binary-Split-Tap-Continue Module (2-STCM)}

\section{The Tap-and-binary-split Switch (Ta2S Switch)}

The binary-split operation is the key of our proposal mainly for two reasons: (a) Realizing binary-split improves the power efficiency, since it divides the incoming power into only two 
outputs; we will show later that, in core networks, this condition may be sufficient and reach a good trade-off between power efficiency and the number of links/wavelengths used; and (b) binary-split is much easier to perform than $n$-split ( $n>2)$, in terms of components used and complexity of fabrication technology.

Therefore, we propose a device with a single input signal and two outputs, which is able to switch to any of the outputs (0:100 or 100:0), or to put the same amount of power to both outputs (50:50). We call this novel device tap-and-binary-split switch (Ta2S switch). Some photonic technologies may be used for this, such as MMIs or Mach-Zehnder Interferometers (MZIs). A possible implementation using MZIs is presented in fig. 3.

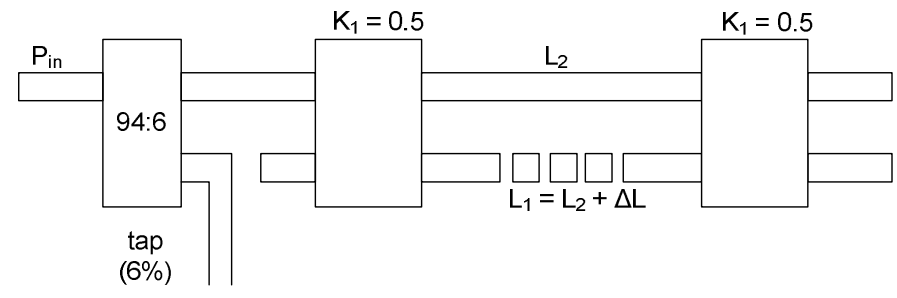

Fig. 3. Block diagram of a Ta2S switch based in Mach-Zehnder Interferometer. $K_{1}$ and $K_{2}$ are coupling ratios and $\Delta L$ is difference of lengths.

The first phase of this device is a fixed tap, which can be made using an MMI coupler. Later there is an MZI optical switch, which can be set up in an initial state in such a manner that both outputs share 50:50 (without causing losses except those due to insertion). By changing the refractive index adequately in the interferometer, the initial condition could be modified to realize the optical switching symmetrically between both outputs.

\section{Block Diagram and Components}

In fig. 4 we present a 4 x 4 2-STC module block diagram. The $P$ inputs go through $P$ Ta2S switches, which will be instructed to perform the operation needed. The resulting optical signals traverse $\log _{2} P+1$ switches instructed to guide them to the desired ports.

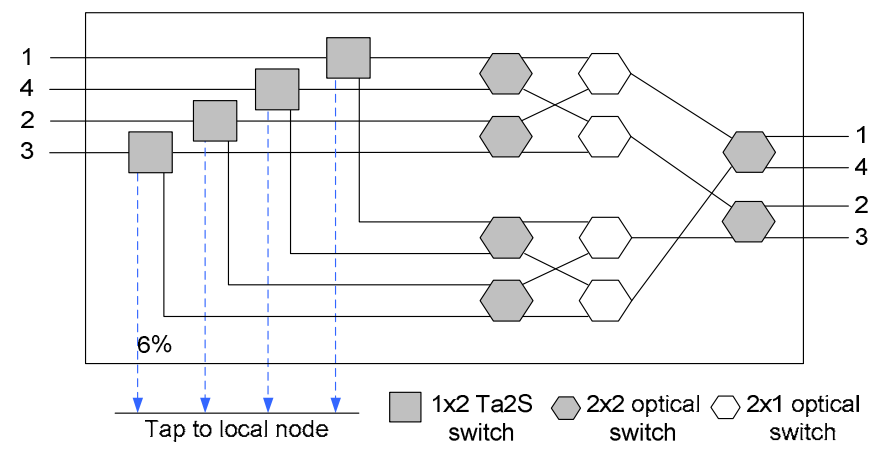

Fig. 4. A 4 × 4 2-STCM.

Note that the optical switch matrix presented here is not similar to any typical $N$ x $M$ crossbar switch matrix (where $N=2 P, M=P$ ) that may be applied in this case. If such a matrix was used, the best case of a switch operation would be given when the signal traverses only one switch, the worst case when it has to traverse $N+M-1$ switches, and the average case, when it traverses $(N+M) / 2$ switches. The important fact to stick out is that the matrix presented here is designed with the aim to use much fewer optical switches, and a signal always traverses the same number of $\log _{2} P+1$ switches.

For a $P \times P / 2$ matrix, if $P$ is a power of 2 , each of the first $\log _{2} P$ phases has $P$ switches and the last one $P / 2$. If it was not the case, a $P^{\prime} \times P^{\prime} / 2$ switch matrix would be given (with $P^{\prime}=2^{\left\lceil\log _{2} P\right\rceil}$ ), and then it should be pruned in order to remove unnecessary switches to get exactly what is needed for $P$ ports. The total number of switches of the switch matrix is presented in table 2, which is much smaller than that of a $P \mathrm{x}$ $P / 2$ matrix.

TABLE I COMPONENTS USED IN INTERNAL MODULES

\begin{tabular}{|c|c|c|c|c|}
\hline & SaD & $\mathrm{TaC}$ & 2-STCM & \\
\hline Optical switches (2x2 / 2x1) & $P^{2}$ & $P^{2}+2 P-1$ & $P \log _{2} P+\frac{P}{2}$ & * \\
\hline Ta2S switches & - & - & $P$ & \\
\hline MMIs & $P(P-1)$ & - & - & \\
\hline Tap devices & - & 1 & $P$ & \\
\hline
\end{tabular}

*This value is stated if $P$ is a power of 2, otherwise it is =

$\left(\log _{2} P^{\prime}\right)\left(2 P-P^{\prime}\right)+\frac{P}{2}$, where $P^{\prime}=2^{\left\lceil\log _{2} P\right\rceil}$

In fig. 5 and table 1 we can see that, unlike $\mathrm{SaD}$ and $\mathrm{TaC}$ modules, our proposal scales conveniently. The total number of components in 2-STCM is much lower and, therefore, a great improvement in power efficiency is achieved, as will be addressed in the next section.

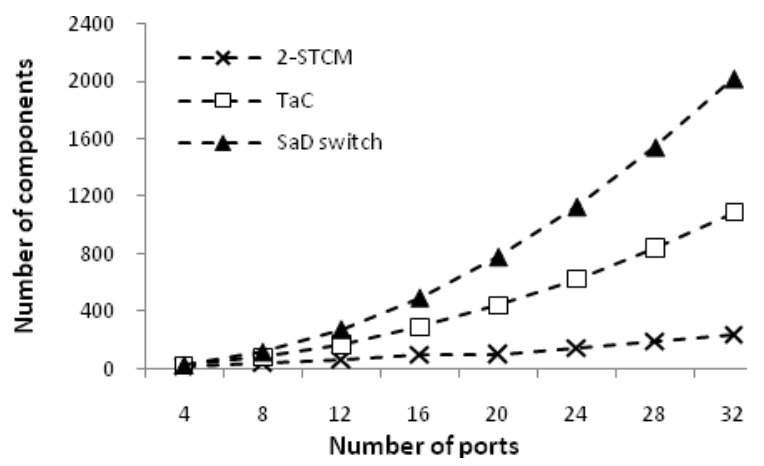

Fig. 5. Comparison of total number of components in 2-STCM, TaC and SaD modules.

\section{EVALUATION OF POWER LOSSES}

With respect to $\mathrm{SaD}$, according to the authors, configurable splitters could be constructed with compact multi-mode interference couplers (MMI) with tunable power splitting ratios, reported in [13]. A $2 \times 2$ MMI coupler is able to tune the output power to different splitting ratios by biasing the refractive index by as little $\Delta n=-0.01$, on the pads specifically located for this purpose. Following simulated results presented 
in [13], a symmetric 2 x 2 MMI does not generate additional losses when output ratios are 50:50 (initial state); but when it is biased in order to change the refractive index and obtain 0:100 or 100:0 split ratios, approximate simulation losses are $1.49 \mathrm{~dB}$ (it actually gets 1:71 and 71:2 splitting ratios). In order to clarify this, in fig. 6 we present a possible implementation of a 1 x 4 configurable splitter. It would be necessary to align $P$ - 1 symmetric 2 x 2 MMI's in cascade, arranged in $\log _{2} P$ phases $(P=$ number of ports). Depending on the action performed, the input power would incur in different losses (see table 2).

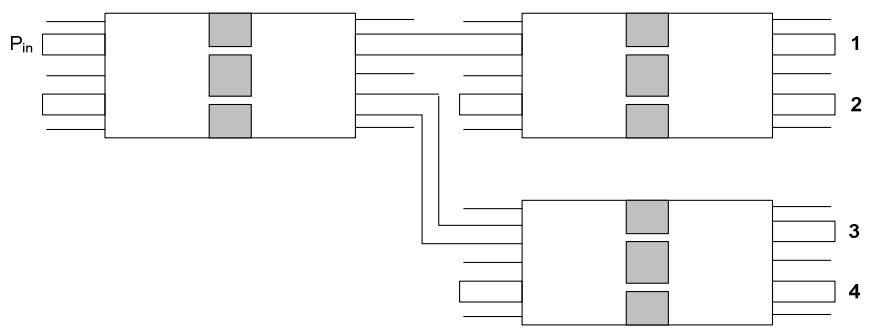

Fig. 6. A possible implementation of a 1 x 4 configurable splitter using 2 x 2 symmetric MMI's with power splitting ratios.

TABLE II

LOSSES (dB) IN CONFIGURABLE SPLITTER OF FIG. 1

\begin{tabular}{lccc}
\hline \hline $\begin{array}{c}\text { Selected output } \\
\text { ports }\end{array}$ & $\begin{array}{c}\text { Loss in } 1^{\text {st }} \\
\text { phase }\end{array}$ & $\begin{array}{c}\text { Loss in } 2^{\text {nd }} \\
\text { phase }\end{array}$ & $\begin{array}{c}\text { Total } \\
\text { loss }\end{array}$ \\
\hline $1,2,3$ and 4 & 0.1 & 0.1 & 0.2 \\
$1,2,3$ or 4 & 1.55 & 1.55 & 3.1 \\
1 and 2 , or 3 and 4 & 1.55 & 0.1 & 1.65 \\
1 and 3, or 2 and 4 & 0.1 & 3.1 & 3.2 \\
$1,2,3$, or $2,3,4$ & 0.1 & 1.55 & 1.65 \\
\hline \hline
\end{tabular}

Losses increase with the number of ports because the number of phases also increases. For instance, for a 1 x 8 configurable splitter, 3 phases will be needed and losses will be in the order of $4.65 \mathrm{~dB}$ for a single switch operation. For a $1 \times 16$ (or $1 \times 12$ ) device ( 4 phases), losses will be in the order of $6.2 \mathrm{~dB}$ for a single switch; and so on. Besides the power losses caused by configurable splitters, a signal has to traverse an optical switch matrix.

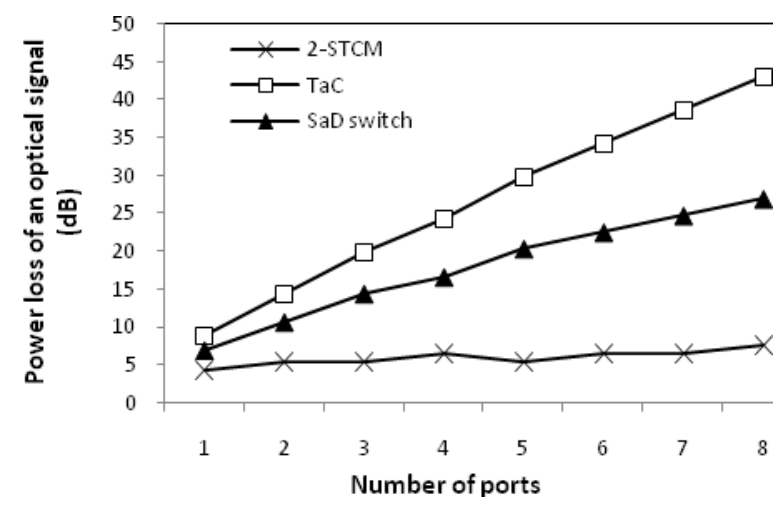

Fig. 7. Comparison of power losses (in $\mathrm{dB}$ ) of a single optical signal for 2STCM, TaC and SaD switch modules when a tap/drop-and-continue action is performed (in the average case).
We shall make an overall assessment of power losses in a network by means of a concrete example. For our calculations we considered that a distributed feedback (DFB) laser at the source node puts out $17 \mathrm{dBm}(50 \mathrm{~mW})$ and the sensitivity of an avalanche photodiode (APD) receiver is $-34 \mathrm{dBm}$ (for 2.5 $\mathrm{Gb} / \mathrm{s}$ ) [14]. Thus, $51 \mathrm{~dB}$ is the power budget of a signal. Although -ideally- amplification can compensate losses, power loss must not exceed the power budget.

We have calculated power losses incurred by a signal when continue, tap-and-continue, binary-split, and tap-and-binarysplit actions are taken in these three modules for the best, average and worst cases. For the purpose of this comparison, no amplification is considered for any node. Let us remember that 2-STCM always taps to the local node (not only when necessary). Because of space limitations, we only present the most significant comparisons, i.e. the average case.

$\mathrm{TaC}, \mathrm{SaD}$ and 2-STCM have been compared in continue and tap-and-continue modes (in the case of $\mathrm{SaD}$, it is a dropand-continue action), and results are presented in fig. 7, for the average case. The estimation is based on the assumption that any optical switch has a power attenuation of $1.1 \mathrm{~dB}$.

$\mathrm{SaD}$ and 2-STCM have been compared in binary-split [see fig. 8 (a)] and tap-and-binary-split modes [see fig. 8 (b)]. Results are presented in the average case; note that in the case of $\mathrm{SaD}$, it is a drop-and-binary-split action. Also notice that both results are slightly different, but it can be observed a clear advantage of 2-STCM. Similar results were obtained for the worst case, but in the best case, losses are almost the same.

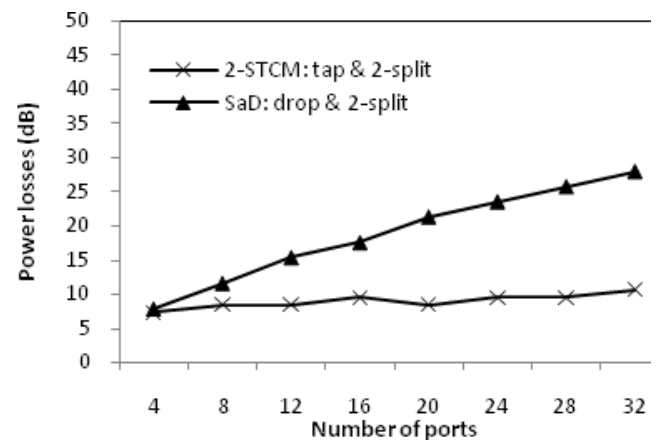

(a)

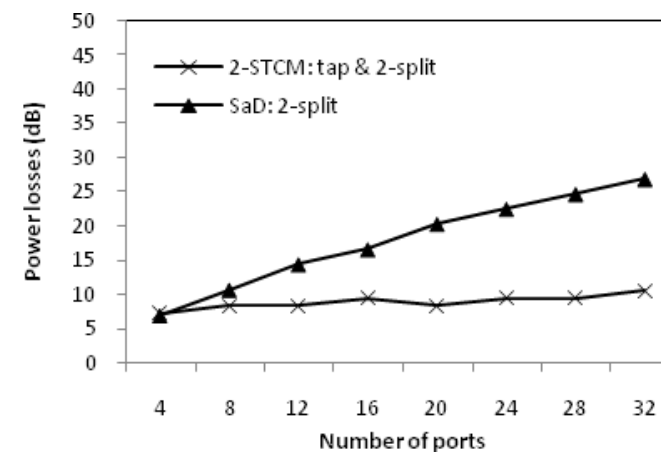

(b)

Fig. 11. Power loss (dB) of an optical signal for 2-STCM and SaD switch when:

(a) A 2-split action is performed

(b) A tap-and-2-split / drop-and-2-split action is performed (both in the average case) 
There is another important issue to mention: if optical amplification inside every node was considered, the 2-STC node is also the most convenient election. It would use much fewer amplifiers $(2 P)$ than $\mathrm{SaD}\left(P^{2}\right)$ but more than TaC $(P)$. However, since best, medium, and worst cases are the same wherever the switching action is performed in 2-STCM, losses may be known in advance and precise amplification levels can be configured beforehand.

\section{Evaluation of Applicability}

It has been shown that 2-STC nodes presents better power efficiency than the other architectures. This is partially due to the 2-STC design but also because in TaC nodes the shared TCM module causes a great cost in terms of losses, because the continue signal requires switching to an output. However, it has to be clearly stated that, when SaD and 2-STC performs splitting, TaC nodes are more power-efficient, because they do not divide the optical signal. The cost that $\mathrm{TaC}$ has to pay is given in the number of links/wavelengths thay need.

One important issue to be studied is if the binary-split condition is enough to satisfy multipoint demands. Since OXC core network nodes do not connect to more than 4 nodes (in general), binary-split should perform well enough. Therefore, we evaluate how well 2-STC nodes may perform on a reference backbone network. We measured metrics and compared them under different multicast requests in a reference network topology: the NSF-Net, which has 14 nodes and 21 fiber links (fig. 9). In the simulations we intentionally excluded the attenuation due to transmission because we focus only on the effect of binary-splitting vs. n-splitting vs. tapping.

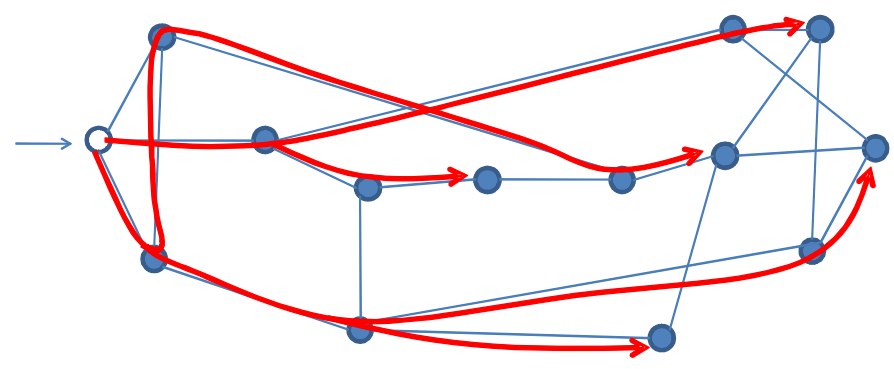

Fig. 9. NSF-Net with an example of a light-tree for the multicast request given. The white node represents the source of the request.

Several light-trees were built by using heuristics to find the minimum Steiner-tree. We measured: (a) the distance from the source to each destination node; (b) the number of links/wavelengths used (bandwidth), and (c) the power ratio per link, which is represented as a percentage (\%) and describes how split and tap actions reduce the 100\% power that is put out at the source node. That concept is similar to the split ratio metric presented in [11]. We have simulated 3 different classes of multicast demands: the first one has 10 members (71\% of total number of nodes), the second one 7 (50\% of total number of nodes), and the third one 4 (28.6\% of total number of nodes). No amplification was considered in theses comparisons.

\section{A. Source-Destinations Distance}

In a TaC-node network, the resulting light-tree actually should be a single lightpath that traverses the minimum number of nodes to reach all destination nodes. In the case of 2-STC nodes, a different light-tree composed of tap-andbinary-split or tap-and-continue actions would be performed. Thus, the network that uses 2-STC nodes would have a better source-destination average distance than a TaC network. Using the same reasoning, SaD-based nodes would perform $n$ split ( $n \geq 2$ ) actions, contributing to decrease the sourcedestination average distance, but decreasing the power ratio per link. We have simulated different shortest-path light-trees for every type of network ( $\mathrm{SaD}, \mathrm{TaC}$ and 2-STC) and results are shown in fig. 10 (a). Note that the 2-STC network improves the power ratio available per link, but it also slightly increases the source-destinations distance, but achieving the desired trade-off.

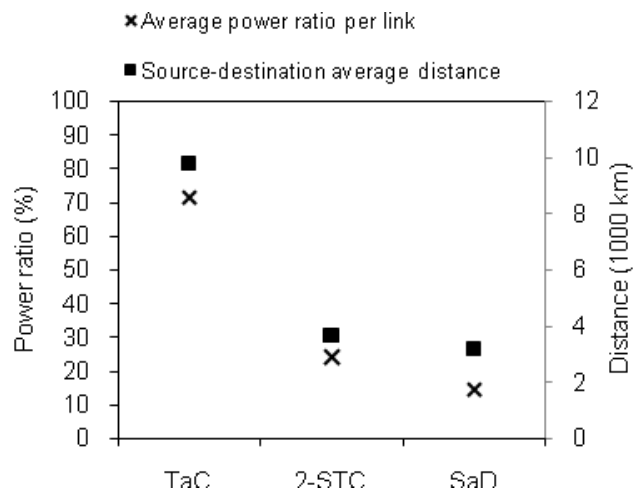

(a)

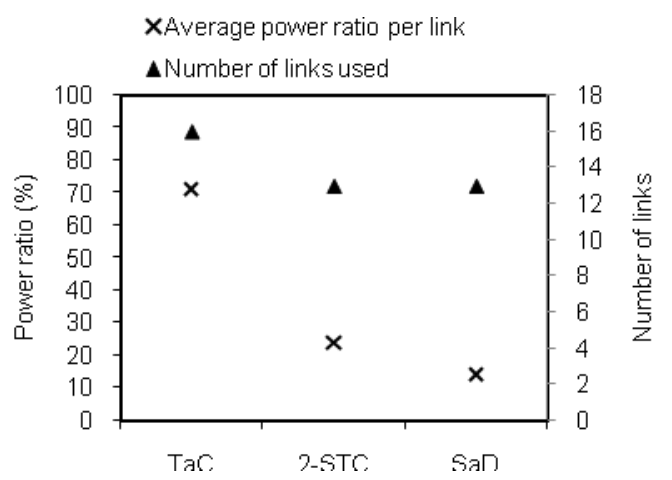

(b)

Fig. 10. Comparison of power ratio, source-destinations distance and number of used links for TaC, 2-STC and SaD networks.

(a) Average power ratio per link (left vertical axis) and average distance source-destination, in units of $1000 \mathrm{~km}$ (right vertical axis).

(b) Average power ratio per link (left vertical axis) and total number of links (right vertical axis).

\section{B. Number of Links/Wavelengths Used}

One important topic to examine is how 2-STC nodes affect the total number of links/wavelengths used. TaC nodes would build a circuit to reach all the members of the multipoint request and hence the lightpath sometimes would have to go 
through some links/wavelengths more than once. In fig. 10 (b), we present a comparison of the average power ratio per link and the number of links/wavelengths employed. We can see that $\mathrm{TaC}$ networks save power, but they spend many more links than 2-STC networks. 2-STC networks save more power than $\mathrm{SaD}$ networks and do not use more links in a significant way.

\section{CONCLUSIONS}

In this paper, a novel multicast-capable OXC node has been proposed called 2-STC, that combines $\mathrm{TaC}$ and $\mathrm{SaD}$ architectures. It performs tap-and-continue and tap-andbinary-split operations to attend multicast requests. This design improves the power efficiency in the tap-and-continue mode with respect to $\mathrm{TaC}$ nodes, and achieves a good tradeoff between use of link resources and power efficiency when used in the tap-and-binary-split mode with respect to both $\mathrm{SaD}$ and $\mathrm{TaC}$ nodes.

The 2-STC node is made up of 2-STCM modules that feature a novel switching matrix in order to improve power efficiency. They permit optical signals to traverse always the same fixed number of optical switches, therefore leading to obtain the same power losses for best, average and worst cases of the optical signals. This has the great advantage to permit to know the required amplification levels beforehand and thus to save in amplification resources, which have an upper limit of usage (i.e. amplification can not be use indiscriminately) Results obtained have shown that the binary-split constraint should be enough to serve any multicast request, at least in a typical reference network topology. As we mentioned previously, it achieves a good balance between the power efficiency and the network link consumption.

\section{ACKNOWLEDGMENT}

The work described in this paper was carried out with the support of the BONE-project ("Building the Future Optical Network in Europe"), a Network of Excellence funded by the European Commission through the 7th ICT-Framework Program. It has also been supported by the Spanish MEC grant TSI2005-07384-C03-02 and PRICIT CCG07-UC3M/TIC3356.

\section{REFERENCES}

[1] Y. Zhou, G-S. Poo. Optical multicast over wavelength-routed WDM networks: A survey. Optical Switching and Networking, Elsevier, 2005.

[2] L. H. Sahasrabuddhe and B. Mukherjee. Light-trees: Optical multicasting for improved performance in wavelength-routed networks. IEEE Communications Magazine, vol. 37, no. 2, Feb. 1999, pp. 67-73.

[3] G. N. Rouskas. Optical layer multicast: Rationale, building blocks, and challenges. IEEE Network, 17 (2003) pp. 60-65.

[4] W. S. Hu and Q. J. Zeng, "Multicasting Optical Cross Connects Employing Splitter-and-Delivery Switch,” IEEE Photonics Tech. Lett., vol. 10, July 1998, pp. 970-72.

[5] M. Ali and J. Deogun, "Power-efficient Design of Multicast Wavelength-Routed Networks," IEEE JSAC, vol. 18, no. 10, 2000, pp. 1852-62.

[6] G. N. Rouskas, "Light-Tree Routing Under Optical Layer Power Budget Constraints," Proc. 17th IEEE Comp. Commun. Wksp., Oct. 14-16, 2002.
[7] M. Ali and J. Deogun, "Cost-efficient Implementation of Multicasting in Wavelength-routed Networks,” IEEE/OSA J. Lightwave Tech., vol. 18, no. 12, Dec. 2000, pp. 1628-38.

[8] H. Zang, J. P. Jue, and B. Mukherjee, "A Review of Routing and Wavelength Assignment Approaches for Wavelength-Routed Optical WDM Networks,” Opt. Net., vol. 1, no. 1, Jan. 2000, pp. 47-60.

[9] F.K. Hwang, D.S. Richards, P. Winter, "The Steiner Tree Problem”, Elsevier, North-Holland, 1992. Annals of Discrete Mathematics, vol. 53.

[10] S. Yan, M. Ali, J. Deogun, "Route optimization of multicast session in sparse light-splitting optical networks", in: Proc. IEEE GLOBECOM, November 2001.

[11] Y. Xin, G. N. Rouskas. Multicast routing under optical layer constraints. IEEE Infocom, vol. 4. 2004, pp. 2731-2742

[12] K.-D. Wu, J.-C. Wu, and C.-S. Yang, "Multicast Routing with Power Consideration in Sparse Splitting WDM Networks," Proc. IEEE ICC, 2001, pp. 513-17.

[13] J. Leuthold and C. H. Joyner, "Multimode Interference Couplers with Tunable Power Splitting Ratios,” IEEE/OSA J. Lightwave Tech., vol. 19, no. 5, May 2001, pp. 700-6.

[14] R. Ramaswami and K. N. Sivarajan. Optical Networks. Morgan Kaufmann Publishers, San Francisco, California, 1998. 